IOS Press

\title{
Erratum
}

\section{Erratum to: Implication of Small Vessel Disease MRI Markers in Alzheimer's Disease and Lewy Body Disease}

Mincheol Park, Kyoungwon Baik, Young-gun Lee, Sung Woo Kang, Jin Ho Jung, Seong Ho Jeong, Phil Hyu Lee, Young H. Sohn and Byoung Seok Ye

[Journal of Alzheimer's Disease, 83(2) (2021), 545-556, 10.3233/JAD-210669]

https://content.iospress.com/articles/journal-of-alzheimers-disease/jad210669

Pre-press 11 January 2022

In the Acknowledgements section on page 562 of the original version of this article, the funding source is acknowledged as 'This study was supported by a Severance Hospital Research fund for Clinical excellence (SHRC) (C2020-0013)'.

However, this study was also supported by a grant of the Korea Health Technology R\&D Project through the Korea Health Industry Development Institute (KHIDI), funded by the Ministry of Health \& Welfare and Ministry of science and ICT, Republic of Korea (grant number : HU20C0511020021).

As such, the correct and updated Acknowledgements section has been placed below.

\section{ACKNOWLEDGMENTS}

The authors are grateful to all the participants who have taken part in this study.

This study was supported by a Severance Hospital Research fund for Clinical excellence (SHRC) (C2020-0013), and by a grant of the Korea Health Technology R\&D Project through the Korea Health Industry Development Institute (KHIDI), funded by the Ministry of Health \& Welfare and Ministry of science and ICT, Republic of Korea (grant number : HU20C0511020021).

Authors' disclosures available online (https:// www.j-alz.com/manuscript-disclosures/21-0669) 